\title{
What's your diagnosis
}

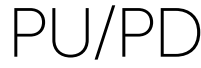

\section{Carmel T. Mooney}

Polyuria and polydipsia (PU/PD) are common complaints in dogs and cats. The well defined list of differential diagnoses lends itself to a logical approach for investigation. Most causes are categorized as primary polydipsia or primary polyuria. Primary polydipsia relates to hypothalamic or behavioural disorders where increased thirst is the main abnormality. These conditions are the least common causes of PU/PD. In primary polyuria, excess urination with compensatory polydipsia is the main abnormality. The causes relate to vasopressin deficiency (central diabetes insipidus), a variety of diseases that influence the kidneys ability to concentrate urine (nephrogenic diabetes insipidus) and those that result in osmotic diuresis. The assignment of individual causes into discrete categories is somewhat artificial as multiple mechanisms may be involved. Although there are other causes of PU/PD, hyperadrenocorticism, diabetes mellitus, hypoadrenocorticism, chronic kidney disease, pyelonephritis, pyometra, hypercalcaemia, liver disease and diabetes insipidus are prioritized in dogs while chronic kidney disease, hyperthyroidism and diabetes mellitus are most pertinent in cats.

The first step in diagnosis is to confirm the existence of PU/PD either by measuring water intake or urine pecific gravity (SG). Consistently low urine SG in the absence of glucosuria is supportive of PU/PD and may allow prioritization of differentials. The signalment, history and physical examination should be evaluated to identify information that aids in refining the differential list. Routine clinical pathological testing allows confirmation of some diseases and may provide evidence for the presence of others that can be confirmed by additional testing. Osmolality can be calculated and may support primary polydipsia. Urine culture is recommended because most animals with pyelonephritis have concurrent cystitis and because urinary tract infections are common with PU/PD. Additional diagnostic tests include symmetrical dimethylarginine, adrenal function tests, bile acid stimulation test, total thyroxine and abdominal ultrasonography, with further more invasive tests (biopsy, pyelocentesis) depending on results.

In some cases, a diagnosis is not achieved despite these investigations. The modified water deprivation test is then performed to distinguish between central diabetes insipidus and primary polydipsia, but only when other causes have been excluded because the test is neither specific nor without risk. Water intake is restricted over 3-5 days. Water is then removed, and the bladder emptied and animal weighed. Water deprivation is continued until a loss of $5 \%$ bodyweight. Haematocrit, total protein, sodium and urea concentrations may also be monitored with urine SG and the test discontinued if marked hypernatraemia ( $>165 \mathrm{mmol} / \mathrm{l}$ ), hyperosmolality (>350 mOsm $/ \mathrm{kg}$ ) or azotaemia occur. An increase in urine SG greater than 1.025 is supportive of primary polydipsia. If urine SG fails to increase adequately, desmopressin is administered intravenously, and the response assessed In central diabetes insipidus, there is little or no increase in urine SG during water deprivation, but a marked increase $>1.010-1.015$ after desmopressin administration. Alternatives to a water deprivation include multiple serial urine SG measurements or a controlled desmopressin trial.

\section{KEY LEARNING OBJECTIVES}

- Be able to refine the differentials for polyuria and polydipsia (PU/PD)

- Define a systematic approach to diagnosis

- Understand the indications, limitations and risk of water deprivation tests

\section{MULTIPLE CHOICE QUESTIONS}

1. A dog is presented with a history of chronic PU/ PD. A urine SG is 1.004. Which of the following diseases is least likely?
(A) Hepatic disease
(B) Hyperadrenocorticism
(C) Chronic kidney disease
(D) Diabetes insipidus

2. A dog is presented with PU/PD. Total calcium is increased at $3.6 \mathrm{mmol} / \mathrm{l}$ (reference interval 2.3-3.0 $\mathrm{mmol} / \mathrm{l})$. Serum parathyroid concentration is $42 \mathrm{pg} / \mathrm{ml}$ (reference interval $20-65 \mathrm{pg} / \mathrm{ml}$ ). How should this be interpreted?

(A) The parathyroid concentration is within reference interval ruling out primary hyperparathyroidism

(B) The combination of high total calcium and reference interval parathyroid hormone concentration is consistent with chronic kidney disease

(C) The parathyroid concentration is inappropriately high for the degree of hypercalcaemia supporting primary hyperparathyroidism

(D) The high total calcium and reference interval parathyroid hormone concentration is consistent with hyperadrenocorticism

3. Which of the following is NOT associated with glucosuria?
(A) Chicken jerky toxicity
(B) Cystitis
(C) Diabetes insipidus
(D) Stress hyperglycaemia 